\title{
Patients' knowledge about their drug allergies
}

\author{
Jonathan P Wyatt
}

\begin{abstract}
Objective-To investigate the nature and accuracy of information carried by patients about their drug allergies. Subjects-2500 new adult patients. Setting-Accident and emergency department of a tertiary referral centre. Methods-Patients were questioned about drug allergies. Where they claimed allergies, general practitioners were contacted for corroboration.

Results-242 patients $(9 \cdot 7 \%)$ claimed 276 allergies; 32 different drugs were implicated. Penicillin was implicated most often (151 patients); 38 patients could not remember what they were allergic to; 21 described severe reactions, but four could not remember the drugs involved. Only seven patients carried evidence of their allergies. General practitioners were contacted about 240 of the drug allergies; only 114 were confirmed as described.

Conclusions-Many patients who believe themselves to have drug allergies are poorly informed about them. Emergency prescribing for these patients may risk anaphylaxis. The future acceptance of "Smart cards" would reduce this risk. In the meantime, patients with drug allergies should be strongly encouraged to carry evidence of their allergies.

( $\mathcal{A}$ Accid Emerg Med 1996;13:114-115)
\end{abstract}

Key terms: drug allergies; anaphylaxis; patient education.

The administration of any drug is potentially hazardous: patients may develop severe and even life threatening reactions. ${ }^{1}$ It has been estimated that fatal anaphylaxis due to penicillin causes between 400 and 800 deaths each year in the United States. ${ }^{2}$ If a patient has previously experienced an allergic reaction to a drug, administration of that drug should be avoided, particularly if an alternative is available. $^{34}$ If no suitable alternative is available, desensitisation has been suggested. ${ }^{35}$

Doctors treating patients for emergency conditions without easy access to their medical records risk prescribing them drugs to which they are allergic, with the attendant possibility of anaphylaxis. This situation occurs frequently in accident and emergency (A\&E) departments and also in general practice outside "normal working hours". In order to minimise the risk, doctors rely upon patients to supply accurate information about their allergies. The aim of this study was to investigate the nature and accuracy of the information carried by patients attending $A \& E$ regarding their allergies.
Methods

Two thousand five hundred adult patients (aged 16 years or over and capable of providing a drug history), seen consecutively by one doctor in $\mathrm{A} \& \mathrm{E}$, were asked about their drug allergies. When patients described a drug allergy, general practitioners (GPs) were contacted to investigate the correlation between information held by them with that provided by the patients themselves.

\section{Results}

Two hundred and forty two patients claimed to have 276 drug allergies, involving 32 different drugs. The most commonly implicated drugs were: penicillin (151 allergies), cotrimoxazole (15), aspirin (12), amoxycillin (9), erythromycin (8), and mefenamic acid (6). Thirty eight patients claimed to have allergies to drugs whose names they could not remember.

The most commonly described reaction was a rash (table 1). Although 57 patients could not recollect the nature of their allergies, 21 described a potentially life threatening reaction (airway swelling, breathing difficulties, or circulatory collapse). Worryingly, four of these patients (including one who had a cardiac arrest) did not know what drug they were allergic to. Only seven of the 242 patients (3\%) carried any evidence of their allergies on them: five carried a written note, two wore a bracelet.

GPs were successfully contacted regarding 240 of the 276 drug allergies. Thirty six drug allergies described could not be investigated in this way, because patients either did not know the name of their GPs or claimed not to be registered (this included four foreign visitors), or because the GPs named had no record of the patients. GPs were unable to confirm the majority of drug allergies as described (table 2).

\section{Discussion}

Almost $10 \%$ of patients attending A\&E in this study claimed to have a drug allergy. As expected, penicillin was implicated most often. ${ }^{6}$ Although reactions are frequently reported by those taking penicillin, true IgE dependent anaphylactic reactions are relatively rare

Table 1 Nature of the 276 "allergies" described

\begin{tabular}{lc}
\hline Reaction & Number \\
\hline Skin rash/blistering & 124 \\
Unknown & 57 \\
Soft tissue swelling & 47 \\
Gastrointestinal disturbance & 12 \\
Breathing difficulties & 6 \\
Dizziness & 6 \\
Lack of efficacy & 6 \\
Other & 16 \\
\hline
\end{tabular}


Table 2 Correlation of 240 described allergies with GP records

\begin{tabular}{lc}
\hline Correlation with GP records & No of allergies \\
\hline Allergy confirmed & $114^{\star}$ \\
Allergy not confirmed: no record of any drug allergy & 97 \\
Allergy not confirmed: records implicate a different drug & 29
\end{tabular}

Allergy not confirmed: records implicate a different drug

${ }^{\star} \mathrm{GP}$ records of nine of these allergies were accompanied by a record of an additional allergy to a different drug, not mentioned by the patient when questioned in this study.

$\left(0.05 \%\right.$ of patients). ${ }^{7}$ In keeping with previous data, it appears that many of the reactions described by patients as "allergies" were not true immunological allergies, but various forms of drug intolerance, or perhaps were even unrelated to the drug. ${ }^{189}$ This may partly account for the poor correlation between patients' reports and information contained within general practitioner records. Although some of these records may be incomplete, it is clear that much of the poor correlation resulted from lack of accurate information held by patients. It is disturbing that 38 patients had forgotten the names of the drugs they believed themselves to be allergic to. This particularly applies to those patients who described severe reactions. Such patients are at risk of anaphylaxis if a drug is given, yet will suffer from untreated illness if no drug treatment is begun. Any patient who suffers an allergic reaction to a drug should be strongly encouraged to carry evidence of this in an information card or bracelet. GPs are in a good position to be able to encourage patients to do this and to help to ensure the accuracy of the information carried.

Recent advances in technology have generated interest in the future possibility that everyone will carry their personal health information in credit card form. ${ }^{10}$ Of course, to be useful, $A \& E$ departments would require appropriate technology to read the cards. Such developments remain controversial, but could bypass the lack of patients' knowledge about their allergies as demonstrated in this study and reduce their risk of receiving inappropriate drugs. ${ }^{10}$

1 Anderson JA. Allergic reactions to drugs and biologica agents. $\mathcal{F} A M A$ 1992;268:2845-57.

2 Errfmeyer JE. Adverse reactions to penicillin. Ann Allergy 1981;47:288-300.

3 Weiss ME. Drug allergy. Med Clin North Am 1992;76: $857-82$.

4 Beeley L. Allergy to penicillin. BMF 1984;288:511-12.

5 Holgate ST. Penicillin allergy: how and when to treat. $B M F$ 1988;296:1213-4.

6 Sher TH. Penicillin hypersensitivity - a review. Pediatr Clin North Am 1983;30:161-76.

7 Idsoe O,Guthe T, Willcox RR, De Weck AL. Nature and dsoe $O, G$ Guthe T, Willcox RR, De Weck AL. Nature and
extent of penicillin side-reactions, with particular extent of penicillin side-reactions, with particular
reference to fatalities from anaphylactic shock. Bull WHO reference to fatalities

8 Kerr JR. Penicillin allergy: a study of incidence as reported by patients. Br $\mathcal{F}$ Clin Pract 1994;48:5-7.

9 Surtees SJ, Stockton MG, Gietzen TW. Allergy to penicillin: fable or fact? $B M F$ 1991;302:1051-2.

10 NHS Management Executive. The care card: evaluation of the Exmouth Project. London: HMSO, 1991. 